\title{
ON THE SO-CALLED PARASITIC PROTOZOA OF CANCER.
}

\author{
By John Lindsay Steven, M.D., and John Brown, M.D.
}

$$
\text { (Plates V. and VI.) }
$$

From the Pathological Laboratory of the Glasgow Royal Infirmary.

Is the following short communication we intend to describe the results of an investigation, which we have recently made upon the question of the presence of parasitic protozoa in cancerous tumours. As the basis of our research, we have taken chiefly the excellent papers on the question recently published by Soudakewitch, and Ruffer and Walker.

Having regard to the full bibliographical and historical introduction to the paper of the latter observers, it is unnecessary for us to dwell upon this aspect of the subject.

It need only be said that, so far as we are able to judge from a somewhat careful study of many of the papers hitherto published, there is a wonderful unanimity as to the description of the bodies, which it has lately been the fashion to denominate coccidia or protozoa. There can be little doubt that, whatever be the opinion of different authors as to the true nature of these bodies, they are generally agreed in their description of their appearances under the microscope, and as modified by the action of different staining reagents. The recent discussion in the Pathological Society of London on Mr. Jackson Clarke's paper elicited a very full expression of opinion from many gentlemen well qualified to speak. From the reports of this discussion we learn that a great many competent observers refuse to recognise the structures described by Jackson Clarke as being in the same category as those usually regarded as parasitic organisms or inclusion bodies by such authors as Soudakewitch, Steinhaus, Ruffer, Galloway, etc.

So far as we can judge from mere written records, we confess to being in sympathy with those who are opposed to the views propounded by $\mathrm{Mr}$. Clarke. At the same time, we are also of opinion that it is quite possible, as was very clearly pointed out by Professor Boyce, to take a different view as to the nature of the bodies described by Ruffer and Walker from that adopted by those workers themselves. 
In the course of our research, our present object has been chiefly to determine whether we could demonstrate, in the ordinary specimens of cancerous formation occurring in our routine work, bodies corresponding in form and structure with those described by previous observers. In the main we have followed the method of preparation recommended by Ruffer and Walker. Many of our preparations have been cut by the Naples method, others embedded in celloidin ; for staining we have employed Biondi's reagent, hæmatoxylin, or a combination of hæmatoxylin and eosin. Many other staining reagents were tried, but the best results were obtained with the help of those just mentioned. We have examined several cases of cancer of the mammæ, two cases of cancer of the stomach, one of cancer of the liver, and one of diffuse cancerous infiltration of the peritoneum and posterior mediastinum. The mammary cancers and one of the gastric cancers, which was removed by operation, were treated by immediate immersion in absolute alcohol, and afterwards by the Naples method. In all these specimens bodies were discovered bearing a close resemblance to the structures described by previous investigators, some of whom regard them as parasitic protozoa, others as due to the endogenous formation of cells.

In what follows it is our intention to base our description of the inclusion bodies chiefly upon the appearances which we observed in two cases of cancerous disease, viz. :-

1. A case of cancerous disease of the pylorus, with secondary gland implication.

2. A case of generalised cancerous infiltration of the peritoneum and mediastinum.

Our reason for this is, that in these specimens the inclusion bodies were remarkable for their extraordinary number, and for the ease with which by staining, either in hæmatoxylin or Biondi, they could be demonstrated.

The first case was that of a rapidly growing cancerous tumour of the pylorus, which had caused considerable dilatation of the stomach, and had been sent in to our colleague, Mr. H. E. Clark, for surgical treatment. In the course of the operation at which one of us (J. L. S.) was present, he removed from the neighbourhood of the pylorus several glands, about the size of marbles, which were at once cut into small pieces, and put into absolute alcohol. On histological examination the cancerous infiltration of the glands was found to present the characters of a typical columnar-celled epithelioma. At some places the arrangement was perfectly regular, and suggestive of tubular glands (Plate V., Fig. 1); at others, the cancer elements were quite irregular, and consisted entirely of heaped-up masses of actively proliferating epithelial corpuscles. In almost every section examined there was no difficulty in recognising numerous bodies having the characters of the so-called parasitic protozoa. These bodies varied in 
many particulars, and a description of the appearances presented will best be given under the following headings:-

Size.-In size there was considerable variation, from a structure somewhat less than a red blood corpuscle to a body, roughly speaking, as large as an ordinary epithelioid corpuscle. Notwithstanding the variation in size the structures were clearly of the same nature, both as regards their reaction to staining reagents and their internal structure.

Situation.-It also soon became apparent that some of the bodies were intracellular, others extracellular (Plate VI., Figs. 4, 5 , and 2). In some cases the extracellular bodies, in their relationships to the surrounding cancer cells, strikingly recall the relations usually seen between the parasites and the proliferating epithelium of the biliary ducts in the rabbit's liver. But whether extracellular or intracellular, the shape of the bodies was almost always perfectly circular, and only occasionally slightly oval. When intracellular in position, the nucleus of the affected corpuscle was frequently found to be pushed to one side, its inner aspect being often concave from the pressure of the inclusion body against it (Plate VI., Figs. 4, 5, 6). Sometimes the nucleus was absent, the whole corpuscle being filled by the "inclusion," and only a thin rim of protoplasm being apparent round its outer wall. In some cases the staining reagent (Biondi) differentiated with almost diagrammatic exactness. The nuclei of the corpuscles took a deep green, the nucleoli red, the protoplasm of the cell-bodies a light brown tint, and the "inclusion" in general a pale sea-green or bluish-green colour. This was particularly the case in the section from which Plate VI., Figs. 7 and 8, were drawn. It is a point worthy of note that in no case did we observe the bodies apart from individual corpuscles or groups of cancer cells. We never found them in the connective tissue stroma; nor were we able to satisfy ourselves of the presence of anything like "inclusions" in the corpuscular nuclei. In reference to this particular case of cancer we were strongly of opinion that the inclusion bodies were most numerous in those areas where the cancerous structure departed most widely from the normal type. Thus, where the tissue was of the character illustrated in Plate V., Fig. 1, the inclusion bodies were almost never seen.

Structure.-The great majority of the inclusion bodies were seen to be possessed of a sharply defined capsule. In many of the Biondi preparations this capsule stained of a brilliant red colour, and appeared like a delicate hair-line surrounding the organism (Plate VI., Fig. 9). In a few instances where the capsule did not present this hair-like red staining the outer portion of the inclusion had a delicate double contour (Plate VI., Figs. 5 and 10). Most of the bodies stained of a delicate sea-green or bluish-green colour, the intensity of the colour being greatest towards the centre of the body. 
Very often there was an absence of colour immediately within the capsule, a feature which has been described by Soudakewitch, and which has been regarded by him as being due to a contraction or shrinkage of the protoplasm of the so-called protozoon. Occasionally the appearances which have been described as a network or series of rays in the protoplasm were observed, but never very definitely, and often leaving the impression that they might be formed as much by the contraction of the protoplasm as by any original structural development in its substance. Sometimes within the delicate hair-like wall of the inclusion a second wavy and granular layer was observed, staining of the same colour as the protoplasm, and forming indeed, one might say, an internal layer of the capsule (Plate VI., Figs. $9 a$ and $9 b$ ).

For the most part the inclusion bodies stain of a greenish or bluish colour throughout, with a concentration of the colouring matter towards the centre, in a few instances so dense and sharply defined as almost to suggest the presence of a green nucleus. The red nucleus of the inclusion body, so beautifully depicted by Metchnikoff in Ruffer and Walker's paper, was not very often seen, perhaps on account of a slight tendency to overstaining, as these authors suppose. Still, in a number of instances, a small round red structure, evidently nuclear, was distictly seen in the midst of the so-called "coccidium," particularly in the section from which Plate VI., Fig. 12, was drawn. With regard to Ruffer and Walker's assertion that leucocytes may wander to the interior of the supposed parasitic bodies, which circumstance is very beantifully illustrated in the plates accompanying their paper, we may call attention to the corpuscle delineated in Plate VI., Figs. 4 and 5 , of the present communication. Here we have a cancer corpuscle containing two inclusion bodies. The larger of these had a concentration of colour towards its central part, in the midst of which was a nucleolar body stained of a deep green colour. Even with the ordinary high power, the structure surrounding this nucleolus was seen to be of a lighter tint; but with the $\frac{1}{18}$ oil-immersion of Zeiss the protoplasm surrounding the nucleolus presented a distinctly orange tint, which at once recalled Metchnikoff's illustrations of leucocyte invasion. This, although the most typical, was not the only corpuscle in which somewhat similar appearances were noted, and in this connection we would also refer to Plate VI., Fig. 11, in which, however, the surrounding layer of orange coloured protoplasm was not observed.

For the most part the intra-corpuscular inclusion bodies were single, but occasionally more than one were noted in the interior of the affected cancer corpuscle. In one corpuscle, in which three inclusions were observed, they were thought to be somewhat smaller than usual, and each of them was possessed of a typical red nucleus. Sometimes one of the bodies was very large, the other much smaller. Multiple inclusion bodies are figured on Plate VI., Figs. 4, 5, and 12.

In some of the affected corpuscles the distinction between corpuscular 
protoplasmic vacuoles and inclusion bodies was made out without very much difficulty (Plate VI., Fig. 8). The vacuoles presented no traces of structure, appearing simply as clear spaces in the brownish-stained protoplasm of the affected corpuscles, and situated, as a general rule, alongside of the inclusions. In Plate VI., Fig. 6, is illustrated a large clear area in the corpuscular protoplasm, which, from the entire absence of all internal structure, we were inclined to regard as a simple vacuole of large size. We have already remarked upon the infrequency with which we met the simple red nuclei in the inclusion bodies, but it should be noted that in some of them numerous brilliant red particles were seen, a circumstance which has also been illustrated for Messrs. Ruffer and Walker by Metchnikoff.

The second case, in connection with which we intend to give a detailed description of the inclusion bodies discovered, was one of diffuse cancerous infiltration of the lymphatic glands of the pelvis, abdomen, and mediastinum, with involvement as well of the abdominal wall and peritoneum. The patient was a young woman, who died with great enlargement of the abdomen and cedema of the lower extremities, the true nature of which during life had been somewhat obscure; a difficulty was even experienced, post-mortem, in tracing the probable source of the cancerous infection, but probably this was situated in the pelvis and most likely in the wall of the urinary bladder, as several rounded cancerous looking nodules of its mucous membrane were present. Portions of the cancer from the pelvis and from the mediastinum were at once placed in absolute alcohol, and afterwards treated by the methods above described. The cancer elements had the characters of very small round epithelial corpuscles with distinct nuclei, and associated with a more or less definite but quite irregularly arranged fibrous stroma. The naked-eye appearances and the general relationship of the disease were more suggestive perhaps of lympho-sarcoma than of cancer, but a very careful consideration of the microscopic characters led to the opinion that the disease was truly cancer.

In the sections from this case the inclusion bodies observed were not so numerous as in the case previously described, and they presented certain differences in character, which are worthy of note. The inclusions were on the whole smaller than those seen in the case of cancer of the pylorus, a circumstance which is probably to be co-related with the fact that the cancer elements themselves were of a very distinctly smaller type. When detected, they were found to be distinctly intracellular in position; and it was at once seen that they did not stain in the same way as those previously described. For the most part, they took a bright red stain throughout, not a pale green or blue tint as in the other specimens. They always presented, also, a quite distinct deeplystained red nucleus. The inclusion bodies in this case are illustrated in Plate VI., Figs. 13, 14, and 15. The corpuscular nuclei stained green, and the protoplasm of the cancer corpuscle orange, the nucleoli being 
red, when Biondi's stain was used. The inclusions were very sharply and strikingly defined. It was noted also that the lymphoid corpuscles of the glandular tissue took a shade of green different from that of the nuclei of the cancer corpuscles - the shade of the lymphoid corpuscles being more of a bright grass green tint. A large number of sections from this case were examined, and, compared with the first, it seemed to us to suggest that different types of cancers might have different types of inclusions.

In what has gone before we have endeavoured to describe the characters of the inclusion bodies, which we have been able to see in our specimens-in some of the cases not without considerable difficulty, but in the two cases particularly referred to, for the most part with comparative ease. It only remains for us to indicate whether we have been able to come to any conclusion as to the true nature of these bodies, which at present are exciting so much attention in pathological circles.

We must frankly confess that, so far as our researches have gone, we are at present unable to formulate any definite conclusion as to the essential nature of these bodies. With reference to a possible biological classification of the inclusions, we are obviously not in a position to make any statement; this is a question which must be left for the skilled judgment of expert zoologists. That the bodies described may be parasitic protozoa we have no reason to deny, because we know that, apart from cancer altogether, such parasites are capable of exciting disease in the human organism. In proof of this we need only refer to the address of Dr. Joseph Coats, delivered from the president's chair of the Glasgow Medico-Chirurgical Society, at the beginning of the present session, and since published in the British Medical Journal. We would also call attention in this connection to the circumstance that dysentery has been definitely proved to be associated with the presence of living amœboid bodies, both in the secretions from the bowel and in the tissues of its wall. Further than this, in reference to the supposed biological nature of the inclusion bodies, we cannot go. The difficulties in arriving at a definite opinion on this interesting subject are greatly enhanced by the fact that hitherto investigators have been quite unable satisfactorily to isolate these structures by cultivation outside of the body.

But apart from this aspect of the question, it might be expected that we should state whether our researches entitle us to form any opinion as to whether the inclusion bodies may or may not be something altogether different from living intracellular parasites. It seems to us that there are mainly two points of view from which the consideration of this question may be taken up-(1) Are the inclusions vacuoles? (2) Are they due to natural degenerative processes, or are they caused by changes in the corpuscles, brought about by the very complicated modes of preparation and staining?

We are of opinion that they are certainly not vacuoles in the proto- 
plasm of the cells, and this for various reasons. Vacuoles would not present the somewhat complicated staining reactions, which we have already described in connection with the inclusion bodies. Further, the size of the bodies, though varying a good deal, is on the whole much more constant than one would expect in connection with simple vacuolated spaces in the protoplasm. The circumstance also that we have seen inclusion bodies and vacuoles in the same corpuscle is in favour of the view that we have now expressed.

As regards the question of these bodies being the products of degenerative changes, we have to say that we have no experience or knowledge of any retrograde metamorphosis, which presents similar characters to them. Occasionally during the examination of a large number of carefully prepared sections, we asked ourselves the question whether it were possible for them to have been produced by the prolonged and complicated processes involved in the preparation of the tissues for examination by the Naples method. That this could hardly be so seemed to us to be proved by the circumstance that we were able to demonstrate the presence of the bodies in sections, which had been hardened in the ordinary way in alcohol. One of us (J. L. S.) has also seen inclusion bodies, presenting generally all the characters described in this paper, in sections from a sarcoma of the mediastinum composed of large spindle or oat shaped corpuscles, and hardened for section cutting in methylated spirit.

The only other hypothesis left for consideration is the old one advanced by Virchow, which would attribute the presence of these peculiar structures to the endogenous formation of cells. That the structures are quite definitely organised, we have no reason to doubt. The manner in which they stain, and the distinct appearances of internal organisation and differentiation which they present, are all in favour of this opinion as to their nature. But, after admitting that they are organised bodies, we must confess that there is, in our opinion, as much evidence for regarding them as endogenously developed cells, as there is for supposing that they are parasites carrying on warfare against the elements of the host. Certainly, in the specimens which we have examined, leucocyte reaction has not been a prominent feature, although a good deal has been made of this by Ruffer and Walker, and although the certain demonstration of its presence would be most important from the point of view of argument by analogy. As has been said, we have seen appearances similar to those described by Ruffer and Walker, as indicative of the invasion of the parasite by leucocytes, but we have not seen it often enough to lay much stress upon it as an element in the argument determining the true nature of the bodies under discussion.

In conclusion, we have to say that we can go no further in the expression of an opinion as to the nature of the inclusion bodies, than to state that they seem to us to be organised elements. 


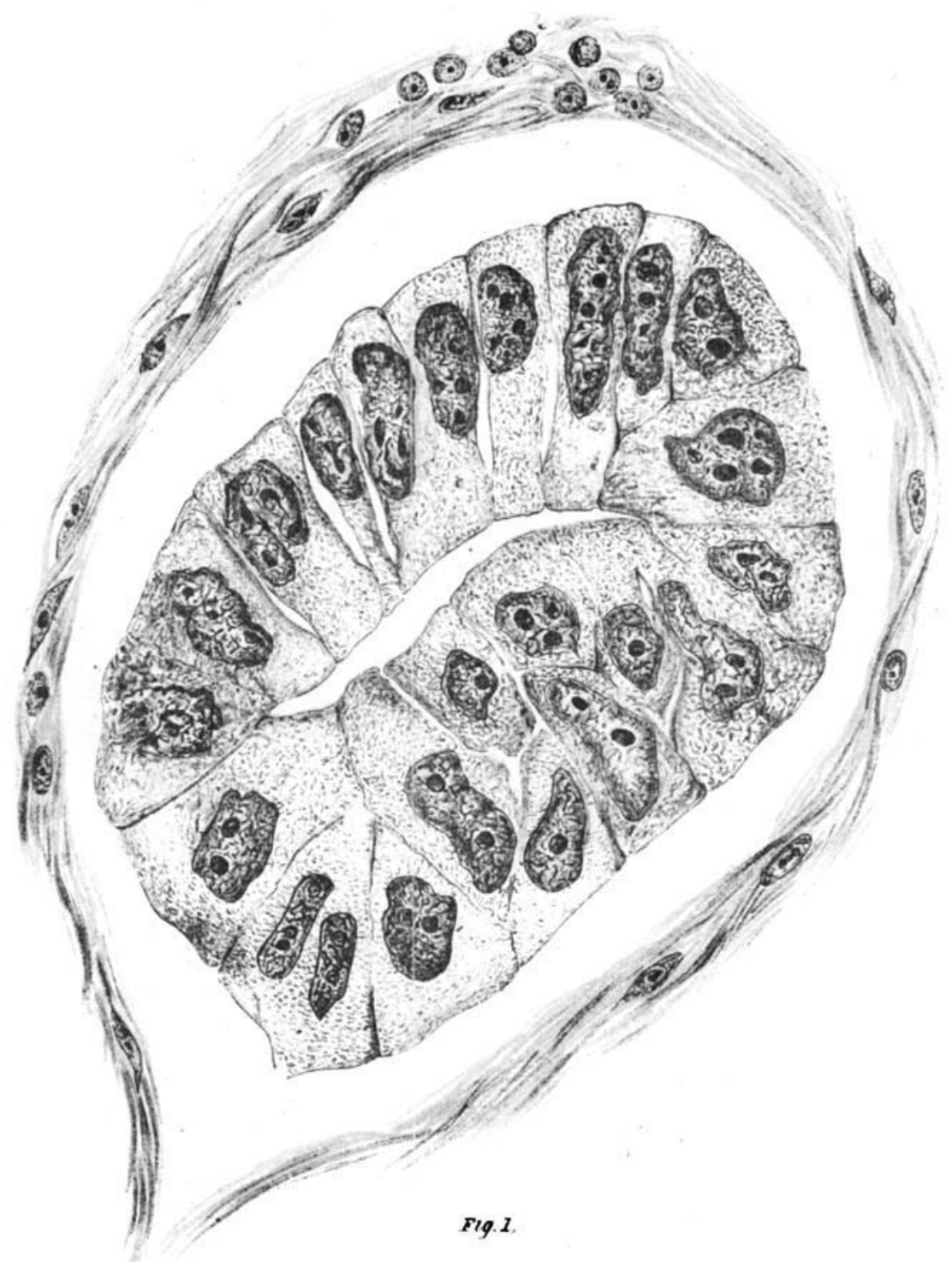




\section{DESCRIPTION OF PLATES.}

\section{Plate V.}

FIG. 1.-Acinus from secondary cancerous infiltration of gland in case of cancer of pylorus. Stained with Biondi's reagent. Oc. 3, and $\frac{1}{18}$ Oil Immersion of Zeiss. Drawn to scale with Zeiss' camera lucida.

\section{Plate VI.}

FrG. 2.-Cancer of pylorus. Gland. Alveolus showing several inclusion bodies, all except one extracellular. Capsule deep red ; protoplasm green and fibrillated. Biondi. Oc. 3, Obj. D. of Zeiss. Drawn to scale.

Fig. 3.-Cancer of pylorus. Gland. Shows two intracellular inclusions. In the lower of the two drawings tracings of the nucleus (corpuscular) are seen, as well as a portion of the stroma. Biondi. Oc. 3, Obj. 3, and camera lucida of Zeiss.

Fig. 4.-Cancer of pylorus. Gland. Cancer corpuscle containing two inclusions, and showing typically the nucleus of the cancer corpuscle. Biondi. Oc. 3, Obj. D. and camera lucida of Zeiss.

Frg. 5.-Cancer of pylorus. Gland. Same corpuscle as shown in Fig. 4, but seen and drawn to scale with Oc. 3 and $\frac{1}{13}$ Oil Immersion of Zeiss (1200 diameters). This corpuscle presented appearances of what is regarded as leucocyte invasion of the parasite.

FIG. 6.-Cancer of pylorus. Gland. Two neighbouring corpusoles showing inclusions. In one of these a large number of deeply stained red particles were visible (they are shown black in illustration). The nucleus seems to have been divided into two by the inclusion. The other, from absence of structure, might be a large vacuole. Biondi. Oc. 3, Obj. Oil Imm. $\frac{1}{15}$. Camera lucida.

Fic. 7.-Cancer of pylorus. Gland. Shows intracellular inclusion, and several neighbouring cancer corpuscles. Biondi. Oc. 3, Obj. D. Camera lucida.

Fia. 8. - Same inclusion as figured in Fig. 7, but drawn with Oc. 3, Obj. Oil Imm. $\frac{1}{18}$, and camera lucida of Zeiss. A small vacuole is seen beside the inclusion, which shows traces of internal structure.

Fic. 9.-Cancer of pylorus. Two extracellular inclusions showing traces of internal structure. Biondi. Oc. 3, Obj. D. Camera lucida of Zeiss.

Fia. 10.-Caneer of pylorus. Gland. Shows an inclusion related to two nuclei, with apparent fusion of the surrounding corpuscular protoplasm. Biondi. Oc. 3, Obj. Oil Imm. $\frac{1}{13}$. Camera lucida of Zeiss.

FIG. 11.-Cancer of pylorus. Gland. An extracellular inclusion, with appearance of leucocyte invasion. Biondi. (a) Oc. 3, Obj. D. (b) Oc. 3, Obj. Oil Imm. $\frac{1}{18}$. Drawn each to scale with camera lucida of Zeiss.

FIG. 12.-Cancer of pylorus. Gland. Showing a group of corpuscles containing inclusions. One shows three small inclusions, each with a red nucleus. Oc. 3, Obj. Oil Imm. $\frac{1}{18}$. Not drawn to scale.

Fia. 13.-Cancerous infection of peritoneum. Shows a small red-stained inclusion. Biondi. Oc. 3, Obj. D. Drawn to scale.

Fig. 14.-Cancerous infection of peritoneum. Shows a small red-stained intracellular inclusion. Biondi. Oc. 3, Obj. Oil Imm. $\frac{1}{18}$. Camera lucida of Zeiss. 
FIG. 15.-Cancerous infection of peritoneum. Alveolus showing several inclusions. Biondi. Oc. 3, Obj. D. Zeiss. Drawn to scale with camera lncida.

Fig. 16.-Cancer of pylorus. Gland. Two cancer corpuscles, containing inclusions, and showing a peculiar honeycombed condition of the corpuscular protoplasm. Biondi. Oc. 3, Obj. Oil Imm. $\frac{1}{18}$. Camera lncida of Zeiss.

Specimens from these cases were shown under the microscope with the oil-immersion lenses at the Meeting of the Glasgow Medico-Chirurgical Society, held on Friday the 5th May 1893 ; and a report of the discussion, which followed the reading of the paper at the same meeting, is to be found in the Glasgow Medical Journal for August 1893. 